\title{
TEMAS DE EDUCAÇÃO EM SAÚDE ABORDADOS NA EDUCAÇÃO BÁSICA ANTES DA PANDEMIA COVID-19
}

\author{
Islana dos Reis Fonseca', Gabriele Marisco da Silva², Benedito Gonçalves \\ Eugenio $^{3}$ \\ ${ }^{1}$ Mestranda no Programa de Pós-Graduação em Ensino da Universidade Estadual do \\ Sudoeste da Bahia (PPGEN/UESB), Vitória da Conquista-BA, Brasil. \\ 2 Prof. ${ }^{\text {a }}$ Titular, Departamento de Ciências Naturais, Universidade Estadual do Sudoeste da \\ Bahia, Vitória da Conquista- BA, Brasil. \\ ${ }^{3}$ Prof. Titular, Departamento de Filosofia e Ciências Humanas da Universidade Estadual do \\ Sudoeste da Bahia, Vitória da Conquista-BA, Brasil. \\ Email para correspondência: islana.fonseca@ftc.edu.br
}

\section{Resumo}

A educação em saúde é um importante campo de conhecimentos e de práticas para promover autonomia, voltadas para a concretização de ações em manter um ambiente saudável e consolidar políticas públicas para a qualidade de vida dos indivíduos. Nesse contexto, emergem as metodologias ativas e aprendizagem como alternativas de complementação de estratégias de ensino, permitindo inferir positivamente no processo educacional. Objetivou-se elencar os temas de educação em saúde abordados na educação básica entre 2014-2019, antes da pandemia de COVID-19. Pesquisa denominada estado da arte realizado por meio do Portal de Periódicos da Capes. Observa-se que os temas sexualidade, saúde escolar, nutrição e saúde bucal são os mais discutidos nas escolas. As principais metodologias utilizadas foram questionários estruturados, atividades práticas, intervenções clínicas, análise e elaboração de material didático. Infere-se a necessidade de proporcionar à comunidade escolar momentos de informação sobre saúde, em diferentes temáticas, incluindo a cientificidade, visto que a pandemia trouxe uma série de discussões sobre a importância da educação em saúde, ainda mais observando a negação da ciência de forma ampla em diferentes setores da sociedade.

Palavras-chave: saúde na escola, ensino e saúde, metodologias ativas.

\begin{abstract}
Health education is an important field of knowledge and practices to promote autonomy, aimed at implementing actions to maintain a healthy environment and consolidate public policies for the quality of life of individuals. In this context, active methodologies and learning emerge as alternatives to complement teaching strategies, allowing for a positive inference in the educational process. The objective was to list the health education topics addressed in basic education between 2014-2019, before the COVID-19 pandemic. State-of-the-art research
\end{abstract}


carried out through the Capes Journal Portal. It is observed that the themes sexuality, school health, nutrition and oral health are the most discussed in schools. The main methodologies used were structured questionnaires, practical activities, clinical interventions, analysis and preparation of teaching material. It is inferred the need to provide the school community with moments of information on health, in different themes, including scientificity, since the pandemic brought a series of discussions about the importance of health education, even more observing the denial of science in a different way. in different sectors of society.

Keywords: school health, teaching and health, active methodologies.

\section{Introdução}

As práticas educativas na promoção de saúde visam desenvolver conhecimentos, habilidades e destrezas para o autocuidado da saúde e a prevenção das condutas de risco em todas as oportunidades educativas; bem como fomentar uma análise sobre os valores, ações, condições sociais e os estilos de vida dos próprios sujeitos envolvidos. No ambiente escolar devem estimular o cidadão para o autocuidado, responsabilizando-o por suas ações, e promovendo sua participação mais ativamente no processo saúde e doença (DUARTE, 2015).

As práticas educativas em saúde na escola, se constituem num processo de formação de escolha do indivíduo por meio da oferta e interação entre o conteúdo teórico e a experiência de vida de cada um e o estabelecimento da confiança e da vinculação do usuário ao serviço de saúde e ao profissional (ALVES; AERTS, 2011).

A educação pública deve ser gratuita, com qualidade, e ofertada indistintamente a jovens e adultos nas regiões onde se inserem. Sendo que 0 cuidado com a saúde de seus estudantes torna-se uma necessidade, como complementação de sua educação e como forma de manutenção de saúde, seja por meio do conhecimento construído e adquirido ou das várias formas de assistência que podem ser oferecidas a eles (ANCINI, 2017).

Os programas de educação em saúde no ambiente escolar, focando os comportamento e hábitos saudáveis dos estudantes, são capazes de melhorar o nível de conhecimento sobre temas relacionados à educação em saúde, 
sendo considerada uma opção efetiva e de baixo custo para a democratização de conhecimentos em saúde (KUBO, 2010).

Caberia, então, aos profissionais da área de saúde a articulação e integração de ações junto ao grupo de educadores, auxiliando na sua implantação, assim como o trabalho junto aos pais e responsáveis na comunidade externa, objetivando o cuidado integral da saúde do estudante, para transformá-lo num ser humano saudável, cidadão consciente e responsável com sua saúde e a da coletividade (BRASIL, 2009).

Diante do atual contexto brasileiro, considerando a pandemia COVID-19, iniciado em 2020 no Brasil, e os desafios apresentados até o momento pela educação, houve o fechamento das escolas de Educação Básica, e o Ministério da Educação (MEC) juntamente com os Conselhos de Educação Nacional e Estaduais propuseram que o atendimento educacional fosse feito de forma remota. O ensino a distância, deu-se com o uso de plataformas como a Google Classroom, ou ainda, redes sociais como Facebook, Instagram, WhatsApp, dentre outras (MONTEIRO, 2020).

As propostas em debate no atual momento são de educação à distância, aulas remotas, plataformas de ensino e são muitas que vem a mobilizar as instituições de ensino em todas as instâncias. Suas propostas metodológicas de interação do professor com o aluno, nomeadas de chats, fóruns, salas de tarefas e uma infinidade de ferramentas disponíveis e suas metodologias (BARBOSA et al., 2020).

Entender que a pandemia do novo coronavírus reposicionou a educação em saúde. As práticas de prevenção e cuidado estão - ou deveriam estar - em um lugar central no cotidiano. A educação traz seu repertório de discussões em relação aos processos de ensino e aprendizagem e, no caso específico, da edificação de outros espaços do conhecimento. A saúde se faz presente como um conceito em mutação, ao se afastar da centralidade na doença, amplia seu foco em olhares multicausais, em que o bem-estar está interligado à nossa condição de seres sociais, no elo entre o humano, a natureza e a sociedade (CHAVES; VALENTE, 2020).

Para os alunos do ensino fundamental atividades de educação em saúde despertam um conhecimento básico de como ter hábitos de higiene com o corpo e do lugar em que frequentam. É possível ressaltar a necessidade de 
iniciativas na escola em trabalhar com a promoção da educação para saúde da criança, e que seja um processo em permanente desenvolvimento. Estes processos devem ser capazes de contribuir para a aquisição de competências das crianças, permitindo-Ihes confrontar-se positivamente consigo mesmas, construir um projeto de vida e ser capazes de fazer escolhas individuais, conscientes e responsáveis (BEZERRA et al., 2016).

Para fundamentar as discussões na escola, o tema da saúde é referenciado em documentos oficiais, com uma abordagem atualizada, para fundamentar a discussão, a BNCC (Base Nacional Curricular Comum) trata sobre os temas que destacam aspectos relativos à saúde, abrindo espaço para discutir o que é preciso para promover a saúde individual e coletiva, inclusive no âmbito das políticas públicas. Além desses, o conhecimento das condições de saúde, saneamento básico, qualidade do ar, condições nutricionais, campanhas de vacinação, programas de saúde da família e comunidade, investimento em pesquisa, campanhas sobre doenças e vetores, no desenvolvimento de condições propícias à saúde (BRASIL, 2017).

Há algum tempo se discute, em documentos oficiais a exemplo das orientações curriculares de Estados e Municípios brasileiros, e mesmo orientações internacionais, a necessidade de se pensar em uma organização do ensino que estimule os alunos a aprender; algumas dessas modalidades fazem parte as "metodologias ativas". A aprendizagem ativa é compatível com uma prática reflexiva, providas atividades que incluam oportunidades de reflexão, como algo que seja parte do próprio processo de aprendizagem ativa, ou seja, refletir acerca da própria aprendizagem (MORAES; CASTELLAR, 2018).

Sendo assim, o processo de ensino-aprendizagem ativo contrasta com o modelo tradicional de ensino, consiste na utilização de técnicas visando favorecer a autonomia do estudante, despertando a curiosidade e estimulando a tomada de decisão individual e coletiva (BORGES; ALENCAR, 2014). Desta forma, ao invés de receber informações de forma passiva, o estudante torna-se o centro do processo ensino-aprendizagem e o responsável pela aquisição de novos conhecimentos. $O$ docente passa a atuar como facilitador e moderador desse processo (BARBOSA; MOURA, 2013). 
Baseada na teoria de Ausubel (2003) ao tratar da aprendizagem cognitiva numa estrutura em constante mutação, sendo que para acontecer à aprendizagem, é necessário organização e integração de informações na estrutura cognitiva do aprendiz, sendo associado ao seu conhecimento prévio.

Aprendizagem significativa trata do processo no qual as condições essenciais envolvem a disposição do aluno para aprender, por ter um papel ativo no processo de ensino aprendizagem, assim como o material didático desenvolvido, deve ser, sobretudo, significativo para o aluno (AGRA et al., 2019).

Deste modo, para que os processos de ensino-aprendizagem sobre educação e saúde sejam efetivos no contexto escolar, o docente deve ser bempreparado e bem-formado pelos cursos de Graduação das universidades. Por consequência, a sensibilização e a formação do corpo docente têm importância fundamental para que a educação em saúde exista de fato (COSTA; GOMES; ZANCUL, 2011).

Objetivou-se elencar os temas de educação em saúde abordados na educação básica entre 2014-2019, antes da pandemia de COVID-19.

\section{Metodologia}

Estudo desenvolvido como "Estado da arte" acerca de temáticas voltadas à saúde no campo educativo foi realizada no o Portal de Periódicos da CAPES/MEC, abrangendo artigos publicados no período de 2014 a 2019, considerando os últimos 5 anos antes do início da pandemia COVID-19.

A pesquisa é caracterizada por identificação, registro e categorização que levem a reflexão e síntese sobre a produção científica de uma determinada área, em um determinado espaço de tempo, congregando periódicos, teses, dissertações e livros sobre uma temática específica (MOROSINI, 2014). O mapeamento foi dividido em quatro etapas, descritas a seguir:

Etapa 1 - Escolha da plataforma: Foi escolhida a plataforma Periódicos Capes e a combinação entre os descritores proporcionou uma seleção interessante dos artigos permitindo assim a execução da proposta metodológica.

Etapa 2 - Escolha dos termos para realização das buscas: a fim de limitar o número de artigos na busca, foram selecionadas palavras-chave 
combinadas que enquadram o foco da pesquisa: "higiene e saúde; na escola; ensino fundamental" e "higiene na escola; ensino fundamental".

Etapa 3 - Leitura dos resumos dos artigos pré-selecionados: para refinar a pesquisa, os resumos de todos os artigos selecionados na etapa anterior foram lidos e selecionados os que realmente tratavam de temas relacionados à educação em saúde na educação básica. Foram incluídos para leitura do resumo, artigos segundo os seguintes critérios de abordagem: verminose; higiene e saúde; revisão histórica em saúde; saúde na escola; zoonoses; metodologias ativas em saúde; sexualidade; saúde bucal; e saúde nutricional. De modo semelhante, excluiu-se os artigos dos quais os temas não estavam relacionados ao pesquisado ou com público-alvo diferente da abordagem escolar. Foram critérios de exclusão artigos que tratavam sobre educação ambiental; estudos antropológicos; impacto ambiental; farmácia universitária; estudo epidemiológico; aspectos climáticos; formação de professores; saúde da mulher; Sistema Único de Saúde (SUS); saúde mental e deficiência intelectual.

Etapa 4 - Leitura dos artigos selecionados: todos os artigos foram lidos na íntegra e seus dados foram sistematizados. Foi realizada a análise por categorização, ou seja, definiu-se as categorias mutuamente exclusivas e homogêneas, com o cuidado em permitir que as categorias esgotassem o conteúdo, e, por fim, realizou-se a classificação de forma objetiva, possibilitando a replicação do estudo (CARLOMAGNO; ROCHA, 2016).

Inicialmente, foram obtidos 24 artigos que se enquadraram na relação da educação em saúde no âmbito escolar, no qual a pesquisa trouxe artigos repetidos nas diferentes combinações dos descritores. Entretanto, considerando com critérios as categorias dos artigos, público alvo destinado, atividades desenvolvidas e compatibilidade com o tema da pesquisa, 19 foram selecionados. O compilado de artigos obtidos por conjunto de descritores está apresentado na Figura 1. 
Artigos identificados por meio da pesquisa na base de dados Periódico Capes utilizando as palavras chaves "Higiene e saúde; na escola; ensino fundamental" $n=179$

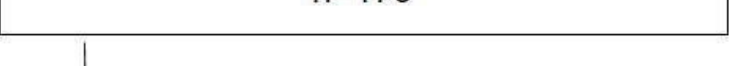

Artigos identificados por meio da pesquisa na base de dados Periódico Capes utilizando as palavras chaves "Higiene na escola; ensino fundamental" $\mathrm{n}=224$ $\rightarrow \quad$ Artigos restantes $\mathrm{n}=12$

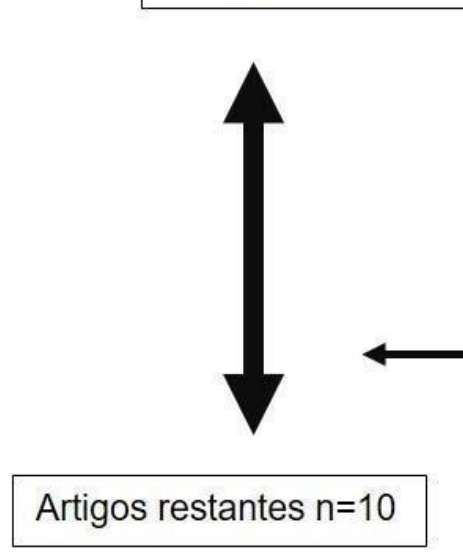

Artigos leitura do texto na integra, considerando categoria dos artigos; público alvo destinado; atividades desenvolvidas; compatibilidade com o tema da pesquisa.
Artigos restantes $n=12$

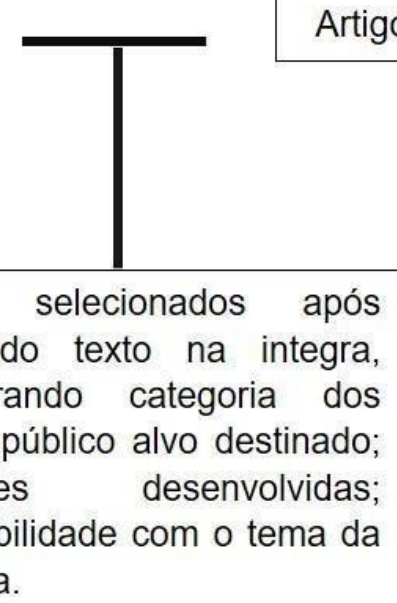

Artigos restantes $n=9$

Figura 1: Compilado dos números de artigos por termos pesquisa na base de dados.

Fonte: Autores

\section{$3 \quad$ Resultados e discussão}

Após a leitura do material selecionado sobre os temas de educação em saúde e higiene abordados na escola, os artigos foram categorizados de acordo com a figura 2. Sendo possível observar que a maioria dos assuntos se referem à sexualidade, seguido das ações em saúde bucal, assuntos relacionados à saúde do escolar e discussões sobre questões alimentares. 


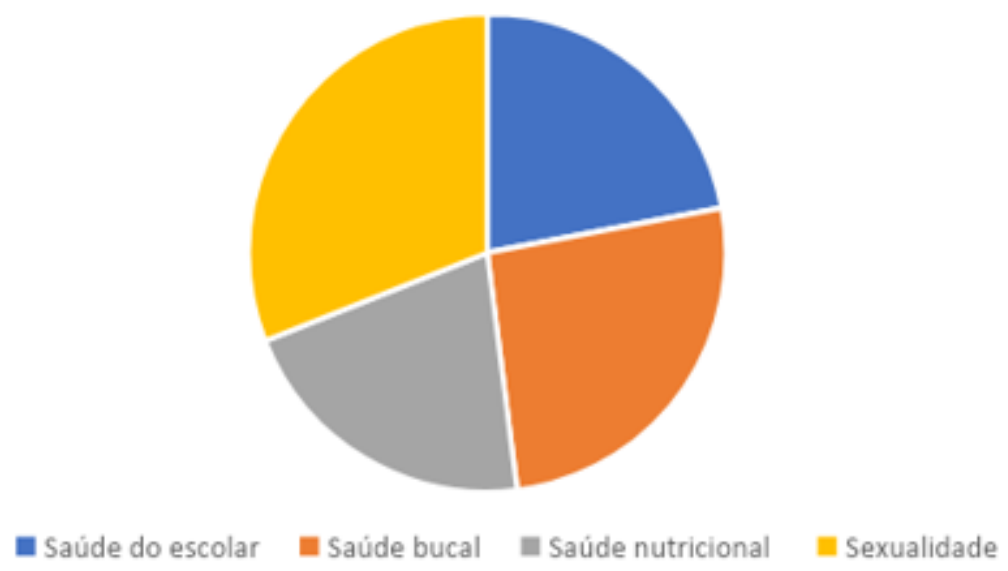

Figura 2: Temas abordados nas escolas sobre educação em saúde.

Fonte: Autores

O Quadro 1 apresenta os temas mais abordados, bem como as metodologias mais utilizadas e o público-alvo ao qual são destinadas às ações desenvolvidas no espaço escolar.

Considerando os temas sexualidade, saúde escolar, nutrição e saúde bucal como sendo os mais abordados no Brasil, destaca-se a importância da amplitude de outras temáticas nas escolas. Como exemplo, trazemos a questão do enfrentamento contra a pandemia do COVID-19. Tanto no ambiente escolar, quanto na ambiência de relações estabelecidas na escola e no ambiente externo devem configurar as ações de promoção da saúde na escola com impacto no ambiente em que se insere a escola, orientações diretas sobre biossegurança e proteção à vida devem ser priorizadas (BRASIL, 2020).

Ressalta-se que há diferenças substanciais entre as famílias, atualmente, em confinamento. Fatores como a quantidade de tempo disponível para se dedicar aos estudos dos filhos; as habilidades não cognitivas dos genitores; a possibilidade de acessar o material online são questões a serem levados em conta quanto ao papel dos pais na educação dos filhos em tempos de pandemia. Toda essa situação gerará um aumento da desigualdade na educação e no progresso do estudante (CIFUENTES-FAURA, 2020).

Nesse contexto, a educação em saúde e ambiente e/ou educação ambiental para promoção da saúde, apresentam temas que exigem interdisciplinaridade e transdisciplinaridade, e a educação em ciências e 
matemática, assumem urgência e centralidade impostas pela própria pandemia (BRASIL, 2020).

\section{Quadro 1: Panorama sobre os temas de educação em saúde nas escolas}

\begin{tabular}{|c|c|c|c|}
\hline Referência & Tema & Metodologia & Público-alvo \\
\hline \multicolumn{4}{|c|}{ Sexualidade } \\
\hline $\begin{array}{l}\text { QUEVEDO; } \\
\text { CONTE, } 2016\end{array}$ & $\begin{array}{c}\text { Violência e } \\
\text { vulnerabilidade social e } \\
\text { afetiva }\end{array}$ & Intervenção psicossocial & $\begin{array}{l}\text { Ensino } \\
\text { fundamental }\end{array}$ \\
\hline HIGA et al., 2015 & $\begin{array}{c}\text { Saúde sexual e } \\
\text { prevenção da gravidez na } \\
\text { adolescência }\end{array}$ & Perguntas abertas & Educadores \\
\hline $\begin{array}{l}\text { ALMEIDA et al., } \\
2014\end{array}$ & $\begin{array}{c}\text { Tratamento e prevenção } \\
\text { do HIV/Aids }\end{array}$ & Questionário & $\begin{array}{c}\text { Ensino } \\
\text { fundamental }\end{array}$ \\
\hline $\begin{array}{l}\text { SALUM; } \\
\text { MONTEIRO, } 2015\end{array}$ & $\begin{array}{c}\text { Doença Sexualmente } \\
\text { Transmissível (DST), } \\
\text { drogas, higiene e } \\
\text { desnutrição }\end{array}$ & $\begin{array}{c}\text { Diagnóstico, questionário } \\
\text { e prática educativa com } \\
\text { abordagem } \\
\text { problematizadora }\end{array}$ & $\begin{array}{c}\text { Ensino } \\
\text { fundamental }\end{array}$ \\
\hline $\begin{array}{l}\text { MIRANDA; } \\
\text { GONZAGA; } \\
\text { PEREIRA; } 2018\end{array}$ & DST & $\begin{array}{c}\text { ABP (Aprendizagem } \\
\text { Baseada em Problemas) } \\
\text { e jogo didático }\end{array}$ & $\begin{array}{c}\text { Ensino } \\
\text { fundamental }\end{array}$ \\
\hline $\begin{array}{l}\text { STEINERT; } \\
\text { HARDOIM; PINTO, } \\
2016\end{array}$ & $\begin{array}{c}\text { Doenças } \\
\text { infectocontagiosas }\end{array}$ & $\begin{array}{c}\text { Ferramentas midiáticas } \\
\text { digitais }\end{array}$ & Ensino médio \\
\hline \multicolumn{4}{|c|}{ Saúde do escolar } \\
\hline $\begin{array}{l}\text { BRAGAGNOLLO et } \\
\text { al., } 2019\end{array}$ & Enteroparasitoses & $\begin{array}{c}\text { Questionário } \\
\text { semiestruturado }\end{array}$ & $\begin{array}{c}\text { Ensino } \\
\text { fundamental }\end{array}$ \\
\hline $\begin{array}{l}\text { SANTOS; LIMA, } \\
2017\end{array}$ & $\begin{array}{l}\text { Parasitoses causadas por } \\
\text { protozoários }\end{array}$ & $\begin{array}{l}\text { Questionário e análise do } \\
\text { livro didático }\end{array}$ & $\begin{array}{l}\text { Ensino médio } \\
\text { (discentes e } \\
\text { docentes) }\end{array}$ \\
\hline $\begin{array}{l}\text { FERNANDES; } \\
\text { PORTELLA; } \\
\text { BASSO, } 2014\end{array}$ & $\begin{array}{l}\text { Pediculose (piolho de } \\
\text { cabeça) }\end{array}$ & $\begin{array}{c}\text { Elaboração de material } \\
\text { didático }\end{array}$ & $\begin{array}{c}\text { Ensino } \\
\text { fundamental }\end{array}$ \\
\hline FELDEN et al., 2016 & Qualidade do sono & Questionário estruturado & $\begin{array}{c}\text { Ensino } \\
\text { fundamental e } \\
\text { médio }\end{array}$ \\
\hline $\begin{array}{l}\text { CASIMIRO et al., } \\
2014\end{array}$ & Saúde escolar & $\begin{array}{c}\text { Revisão bibliográfica } \\
(1995-2012)\end{array}$ & $\begin{array}{l}\text { Crianças em } \\
\text { idade escolar }\end{array}$ \\
\hline \multicolumn{4}{|c|}{ Nutrição } \\
\hline ALVES et al., 2014 & $\begin{array}{l}\text { Aspectos nutricionais } \\
\text { (hipertensão) }\end{array}$ & Questionário & $\begin{array}{c}\text { Ensino } \\
\text { fundamental }\end{array}$ \\
\hline $\begin{array}{l}\text { PARDINO et al., } \\
2019\end{array}$ & Educação alimentar & $\begin{array}{c}\text { Oficinas sobre } \\
\text { alimentação saudável }\end{array}$ & $\begin{array}{c}\text { Ensino } \\
\text { fundamental }\end{array}$ \\
\hline SALES et al., 2014 & Hábitos alimentares & Questionário & 15 a 19 anos \\
\hline $\begin{array}{l}\text { SANTOS et al., } \\
2014\end{array}$ & Hábitos alimentares & Horta escolar & $\begin{array}{c}\text { Ensino } \\
\text { fundamental }\end{array}$ \\
\hline \multicolumn{4}{|c|}{ Saúde bucal } \\
\hline $\begin{array}{l}\text { BEZERRA; GOES, } \\
2014\end{array}$ & Saúde bucal & Questionário & 15 a 19 anos \\
\hline $\begin{array}{l}\text { VAZQUEZ et al., } \\
2015\end{array}$ & Saúde bucal (cárie) & $\begin{array}{c}\text { Questionário + } \\
\text { intervenção }\end{array}$ & $\begin{array}{c}\text { Ensino } \\
\text { fundamental }\end{array}$ \\
\hline $\begin{array}{l}\text { FILGUEIRA et al., } \\
2016\end{array}$ & Saúde bucal & $\begin{array}{c}\text { Questionário + exame } \\
\text { clínico }\end{array}$ & 15 a 19 anos \\
\hline $\begin{array}{l}\text { BORGES et al., } \\
2016\end{array}$ & $\begin{array}{l}\text { Saúde bucal (não adesão } \\
\text { ao tratamento) }\end{array}$ & $\begin{array}{l}\text { Entrevista roteiro } \\
\text { semiestruturado }\end{array}$ & 15 a 19 anos \\
\hline
\end{tabular}


Ressalta-se que poucos estudos que abordam educação em saúde no ensino médio (31\%), neste contexto, as escolas podem buscar como apoio o Programa Saúde na Escola, criado em 2007 em parceria com Ministério da Saúde e da Educação, com intuito de promover, avaliar a saúde e prevenir doenças dos estudantes. Integra ao programa ações voltadas para os temas combate ao Aedes aegypti, promoção da segurança alimentar, nutricional, da alimentação saudável e combate a obesidade; prevenção de DST/AIDS; prevenção ao uso de álcool e drogas; promoção da atividade física e do lazer nas escolas; prevenção das violências e acidentes (MOREIRA, 2020).

O ambiente escolar deve ser um espaço promotor de saúde, criando relações dialógicas que devem assegurar a educação global, sistêmica, reflexiva, crítica e criativa, desenvolvendo habilidades e atitudes que valorizam a vida. A escola promotora da saúde visa o ensino para o desenvolvimento da cidadania e pretende ser colaborativa, reconectada com a comunidade escolar, valorizando a voz de todos os integrantes do sistema escolar, sendo, portanto, uma escola que oferece diálogo de saberes (BRASIL, 2020).

Percebe-se que ao analisar os artigos selecionados, houve limitação de metodologias por parte dos autores, visto que $58 \%$ dos dados obtidos referemse à aplicação de questionário como forma de mensurar o conhecimento dos alunos sobre os temas de educação em saúde (ALVES et al, 2014; BEZERRA; GOES, 2014; SALES et al., 2014; VAZQUEZ et al., 2015; FELDEN et al., 2016; FILGUEIRA et al., 2016; SANTOS; LIMA, 2017; BRAGAGNOLLO et al., 2019 ).

Em contrapartida, apenas $26 \%$ utilizou metodologias ativas como forma de intervenção, o que representa um número reduzido diante das possibilidades de recursos metodológicos existentes que impactam numa aprendizagem significativa (FERNANDES; PORTELLA; BASSO, 2014; SANTOS et al, 2014; VAZQUEZ et al, 2015; PARDINO et al, 2019;).

Pesquisa realizada sobre metodologias ativas de ensino e aprendizagem, concluíram que estas trazem benefícios gigantescos aos estudantes, tais quais: o desenvolvimento da autonomia do aluno, o rompimento com o modelo tradicional, o trabalho em equipe, a integração entre teoria e prática, o desenvolvimento de uma visão crítica da realidade e o favorecimento de uma avaliação formativa (SOUZA et al., 2020). 
$\mathrm{Na}$ aprendizagem significativa, a informação recebida precisa se relacionar com as informações que o indivíduo já possui, e deste modo o conhecimento recebido não será apenas memorizado, mas será assimilado e terá significado, tornando a aprendizagem efetiva (VALADARES, 2011).

No cenário educacional, as metodologias ativas são ótimas estratégias para a construção do conhecimento e desenvolvimento da aprendizagem significativa. Em contraste com o modelo de ensino tradicional, a abordagem realizada com metodologias ativas ressalta a importância do aluno dentro dos processos de ensino e aprendizagem, com o foco na investigação, resolução de problemas e na descoberta, desenvolvendo o senso crítico e promovendo interação entre professores e colegas (VALENTE, 2018).

A busca de metodologias inovadoras que superem o modelo tradicional de ensino tem sido um desafio no campo educacional. As metodologias ativas são estratégias que estimulam nos alunos a ação-reflexão-ação, fazendo com que eles desenvolvam o aprendizado por meio de experiências, resolução de problemas e outras ações motivadoras (GEMIGNANI, 2013).

$\mathrm{Na}$ escola, contudo, o modo como a abordagem de temas relativos à saúde fica basicamente centrado na transmissão de informações sobre como as pessoas adoecem, os principais ciclos, sintomas, formas de prevenção e controle das doenças (JESUS; SAWITZKI, 2017; JESUS; GARCIA, 2018).

Nessa perspectiva, surgem outras ações como as de avaliação das práticas promotoras de saúde na escola de modo mais abrangente, incluindo 0 ambiente escolar e suas condições como elemento de análise, utilizando variadas formas de coleta de dados, como o questionário, e adaptando às necessidades e características próprias de suas localidades ou regiões e aos interesses dos alunos (PINTO et al., 2016).

\section{Considerações finais}

Neste estudo, foi possível identificar que nos anos de 2014-2019, período que antecede a pandemia COVID-19 os principais temas abordados nas escolas sobre saúde, são referentes à sexualidade, medidas profiláticas sobre a saúde do aluno, aspectos nutricionais e saúde bucal.

A pluralidade de conteúdos existentes em saúde e as necessidades de um levantamento sobre as fragilidades nesta área de ensino se faz necessário, 
visto que os assuntos relacionados à saúde são de extrema relevância para serem abordados no âmbito escolar e não podem ser negligenciados.

Infere-se a necessidade de proporcionar à comunidade escolar momentos de informação, na intenção de ampliar as discussões sobre a saúde, em diferentes temáticas, incluindo a cientificidade relacionando com os cuidados de saúde. Considerando que a pandemia trouxe uma série de discussões sobre a importância da educação em saúde, em todos os níveis de ensino, ainda mais a negação da ciência de forma ampla em diferentes setores da sociedade.

\section{Referências}

AGRA, et al. Análise do conceito de Aprendizagem Significativa à luz da Teoria de Ausubel. Rev. Bras. Enferm., Brasília, v. 72, n. 1, p. 248-255, Feb. 2019. Disponível em: $<$ http://www.scielo.br/scielo.php?script=sci_arttext\&pid=S003471672019000100248\&lng=en\&nrm=iso $>$. Acesso em: 02 Mai 2021. https://doi.org/10.1590/0034-7167-2017-0691.

ALVES, G. G.; AERTS, D. As práticas educativas em saúde e a Estratégia Saúde da Família. Ciência \& Saúde Coletiva, v. 16, n.11, p. 319-325, 2011.

ALVES, M. J. Q. F. A. Aspectos nutricionais ligados à hipertensão: estudo exploratório desenvolvido no ensino fundamental da rede pública de Botucatu. Revista Ciência em Extensão. Vol.10. n 2, 2014.

ANCINI, D.M.B. Implantação de ações de educação em saúde no Instituto Federal Farroupilha campus Alegrete integradas ao programa saúde na escola. Dissertação (Mestrado) - Universidade Federal do Rio Grande do Sul, Faculdade de Medicina, Programa de Pós-Graduação em Ensino na Saúde, Porto Alegre, BRRS, 2017.

AUSUBEL, D. P. Aquisição e retenção de conhecimentos: uma perspectiva cognitiva. Lisboa: Plátano, v. 1, 2003.

BARBOSA, E.F.; MOURA, D. G. Metodologias ativas de aprendizagem na educação profissional e tecnológica. Boletim Técnico Senac. v.39, n.2, p.48-67, 2013.

BARBOSA, A.M. et al. Aulas presenciais em tempos de pandemia: relatos de experiências de professores do nível superior sobre as aulas remotas. Rev. Augustus. Rio de Janeiro, v.25, n. 51, p. 237- 254, jul./out. 2020.

BEZERRA, S.F. et. al. Educação e saúde na escola com ênfase em higiene pessoal e coletiva no ensino fundamental $1.7^{\circ}$ Congresso Brasileiro de Extensão Universitária. $\quad$ Set. 2016.2 Disponível em:https://cbeu.ufop.br/anais_files/905ba915da1392689110732fc3fa1d05.pdf Acesso em: 20 jan. 2021.

BEZERRA, I. A.; GOES, P. S. A. Associação entre capital social, condições e comportamentos de saúde bucal. Ciênc. saúde coletiva, Rio de Janeiro, v. 19, n. 6, p. 1943-1950, jun. $2014 . \quad$ Disponível em: 
<http://www.scielo.br/scielo.php?script=sci_arttext\&pid=S1413-

81232014000601943\&lng=en\&nrm=iso $>$. Acesso em: 02 Mai 2021. https://doi.org/10.1590/1413-81232014196.06242013.

BORGES, T.S.; ALENCAR, G. Metodologias ativas na promoção da formação crítica do estudante: o uso das metodologias ativas como recurso didático na formação crítica do estudante de ensino superior. Cairu em Revista. n.4, p. 119-143, 2014.

BRAGAGNOLLO, G. R. et al. Intervenção educativa lúdica sobre parasitoses intestinais com escolares. Rev. Bras. Enferm., Brasília, v. 72, n. 5, p. 1203-1210, Cot. 2019. Disponível em: <http://www.scielo.br/scielo.php?script=sci_arttext\&pid=S003471672019000501203\&lng=en\&nrm=iso $>$. Acesso em: 02 Mai. 2021. Epub Sep 16, 2019. https://doi.org/10.1590/0034-7167-2017-0551.

BRASIL. Base Nacional Comum Curricular (BNCC). Brasília: MEC. 2017. Disponível em: <http://basenacionalcomum.mec.gov.br/images/BNCC_20dez_site.pdf > Acesso 20 maio 2019.

BRASIL. Nota técnica n. 1/2020/PG-EBS/IOC-FIOCRUZ. Embasamento técnico e sugestões para ações de promoção da saúde ambiental e estratégias educacionais para mitigar as iniquidades no acesso à Educação Básica no Brasil no contexto da pandemia de COVID-19. jul. 2020.

BRASIL. Saúde na escola. Série B. Textos Básicos de Saúde. Cadernos de Atenção Básica; n. 24, Ministério da Saúde, Secretaria de Atenção à Saúde, Departamento de Atenção Básica Brasília: Ministério da Saúde, 96 p.: il 2009.

CARLOMAGNO, M. C.; ROCHA, L. C. Como criar e classificar categorias para fazer análise de conteúdo: uma questão metodológica. Revista Eletrônica de Ciência Política, vol. 7, n. 1, 2016.

CHAVES, B.S.; VALENTE, P.A. A Educação em Saúde em tempos de pandemia: tecnologias e novos sentidos no campo. Pensar a educação em pauta. Um jornal para a educação brasileira. Ano 8, n. 283, julho de 2020.

CIFUENTES-FAURA, J. Consecuencias en los niños del cierre de escuelas por Covid19: el papel del gobierno, profesores y padres. Revista Internacional de Educación para la Justicia Social, Madrid, v. 9, n. 3e, p. 1-12, 2020. Disponível em: https://revistas.uam.es/riejs/article/view/12216/12089. Acesso em: 20 jan. 2021.

COSTA, S.; GOMES, P. H. M.; ZANCUL, M. S. Educação em saúde na escola na concepção de professores de Ciências e de Biologia. ENCONTRO NACIONAL DE PESQUISA EM EDUCAÇÃO EM CIÊNCIAS, 8., 2011, Campinas. Anais Campinas: Associação Brasileira de Pesquisa em Educação em Ciências, 2011.

DUARTE, A. P. Práticas educativas em saúde no ambiente escolar: uma proposta de intervenção. Trabalho de Conclusão de Curso apresentado ao Curso de Especialização em Estratégia Saúde da Família, Universidade Federal do Triângulo Mineiro, Uberaba, 2015.

FELDEN, E. P. G. et al . Adolescentes com sonolência diurna excessiva passam mais tempo em comportamento sedentário. Rev Bras Med Esporte, São Paulo, v. 22, n. 3, p. 186-190, June 2016 Disponível em: <http://www.scielo.br/scielo.php?script=sci_arttext\&pid=S1517-

86922016000300186\&lng=en\&nrm=iso $>$. Acesso em: 02 May 2021. https://doi.org/10.1590/1517-869220162203147290. 
FERNANDES, M.; PORTELLA, R. O.; BASSO, S. P.S. Higiene pessoal na perspectiva da educação inclusiva. Revista Eletrônica de Educação, v. 8, n. 3, p. 266-277, 2014. 2014

FILGUEIRA, Ana Cristina Gondim et al. Saúde bucal de adolescentes escolares. Holos (Natal. Online), v. 1, n. 1, p. 161, 2016.

GEMIGNANI, E. Y. M. Y. Formação de professores e metodologias ativas de ensinoaprendizagem: ensinar para a compreensão. Fronteiras da Educação, v. 1, n. 2, 2013.

JESUS, R. F.; GARCIA, R. N. Abordagem de temas referentes à saúde no ensino de biologia: a perspectiva de professores da área e em documentos escolares. Revista Eletrônica Científica Ensino Interdisciplinar. Mossoró, v. 4, n. 12, 2018.

JESUS, R. F.; SAWITZKI, R. L. Formação de professoras unidocentes e o tema transversal saúde: possibilidades e apontamentos. Revista Electrónica de Enseñanza de las Ciencias, v. 16, n. 2, p. 341-361, 2017.

KUBO, F. M. M. O professor e a educação em saúde: um estudo qualiquantitativo. Tese (Doutorado) - Universidade Estadual de Campinas, Faculdade de Odontologia de Piracicaba. Piracicaba, SP: [s.n.], 2010.

MORAES, J.V.; CASTELLAR, S.M.V. Metodologias ativas para o ensino de Geografia: um estudo centrado em jogos. Revista Electrónica de Enseñanza de las Ciencias, v.17, n.2, p.422-436., 2018.

MOROSINI, M. C.; NASCIMENTO, L. M. Internacionalização da educação superior no brasil: a produção recente em teses e dissertações. Educ. rev., Belo Horizonte, v. 33, e155071, $2017 . \quad$ Disponível em: <http://www.scielo.br/scielo.php?script=sci_arttext\&pid=S0102-

46982017000100109\&lng=en\&nrm=iso>. Acesso em: 07 jul. 2020.

MONTEIRO, S.S. (Re) inventar educação escolar no Brasil em tempos da COVID-19. Rev. Augustus. Rio de Janeiro, v.25, n. 51, p. 237- 254, jul./out. 2020.

MOREIRA, D. Secretaria Municipal de Educação de São José. Portal da Educação. Disponível em: https://saojose.educarweb.net.br/portal/demo/\#/saude-home. Acesso em: jan. 2021.

PINTO, R.O. et al. Validação de instrumento desenvolvido para avaliação da promoção de saúde na escola. Rev. Saúde Pública. 2016; 50:2.

SALES, F. H. S.; et al. Maus Hábitos Alimentares de Estudantes do Ensino Médio em Escolas Públicas. Holos, vol. 4, 2014, pp. 502-511 Instituto Federal de Educação, Ciência e Tecnologia do Rio Grande do Norte

SANTOS, L. M.; LIMA, N. B. Análise da abordagem e conhecimento do tema parasitoses causadas por protozoários em escolas públicas do município de SalinasMG. ACTA Biomedica Brasiliensia. Vol.8. n 2. Dez. 2017.

SANTOS, M. J. D. et al. Horta escolar agroecológica: incentivadora da aprendizagem e de mudanças de hábitos alimentares no ensino fundamental. HOLOS, [S.I.], v. 4, p. 278-290, set. 2014. ISSN 1807-1600. Disponível em: <http://www2.ifrn.edu.br/ojs/index.php/HOLOS/article/view/1705>. Acesso em: 02 maio 2021. doi:https://doi.org/10.15628/holos.2014.1705. 
SOUZA, A. L. A.; VILAÇA, A. L. A.; TEIXEIRA, H. J. B. Os benefícios da metodologia ativa de aprendizagem na educação p.32-47. In: Metodologias ativas: métodos e práticas para o século XXI / Gercimar Martins Cabral Costa (Organizador). Quirinópolis, GO: Editora IGM, 2020.

VALADARES, J. A teoria da aprendizagem significativa como teoria construtivista. Aprendizagem Significativa em Revista, v. 1, n. 1, p. 36-57, 2011.

VALENTE, J. A. A sala de aula invertida e a possibilidade do ensino personalizado: uma experiência com a graduação em midialogia. Metodologias ativas para uma educação inovadora: uma abordagem teórico-prática. Porto Alegre: Penso, p. 2644, 2018. 